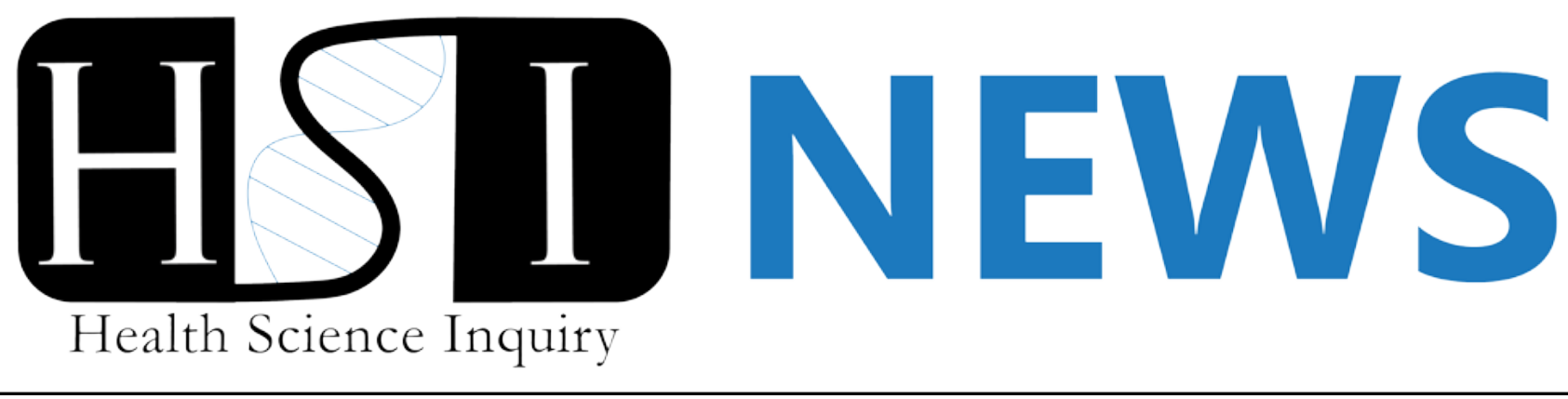

\title{
Artificial intelligence and mental illness
}

\author{
-Kevin Dick
}

\begin{abstract}
$\mathbf{T}$ echnology has become increasingly present in all corners of our lives. We leave a trail of our digital selves in the wake of our interactions with smart phones, smart devices, social media, and the Internet. Our digital signature provides unprecedented access to the unseen side of human behavior and has increasingly become a viable source of data for research into human health. Furthermore, with the digitalization of medical records, artificial intelligence and machine learning $(\mathrm{Al} / \mathrm{ML})$ have been adopted to develop predictive models, clinical decision support systems, and synthesize information from disparate sources. Recently having entered the proverbial Al summer, a period of rapid development and considerable funding, $\mathrm{Al} / \mathrm{ML}$ applications have become increasingly present in research. Numerous fields are applying $\mathrm{Al} / \mathrm{ML}$ algorithms in their work, and healthcare stands to benefit substantially from its widespread adoption. Researchers throughout Canada are addressing a broad range of chronic diseases and illnesses with $\mathrm{Al} / \mathrm{ML}$ as a primary tool.
\end{abstract}

A key trend in contemporary healthcare has been the emergence of corporate entrants such as IBM, Microsoft, Apple, Facebook, and Alphabet Inc. [1, 2]. Chronic depression and other mental illnesses have been identified as one example of benefitting from the appraisal of the plethora of social media data available for detection and preventative measures. The Ottawa-based company, Advanced Symbolics Inc. (ASI), has recently been contracted by the Canadian Federal Government on a three-month pilot project leveraging social media data to detect suicide-related behavior [3]. Cofounder and Chief Scientist Dr. Kenton White, an adjunct professor of Computer Science at the University of Ottawa, is spear-heading the initiative and ASI will be focusing on suicide-related behavior and measuring ideation (i.e. thoughts), behaviors (i.e. self-harm), and communications (i.e. suicidal threats, plans of suicide). With the use of its patented Al technology Polly, ASI will use randomized, controlled samples of 160,000 Canadians in its representative sample. Polly is trained to learn the patterns using a large labeled corpus for a specific subject or issue and will look to identify and correlate patterns in the segments of the Canadian population who discuss suicide-related behavior based on publicly available but non-personally identifiable online data. When interviewing a representative of ASI about major challenges to this work, privacy concerns were highlighted:

"Privacy is of extreme importance to ASI. To maintain privacy, we anonymize our data. All data is taken from public online sites where each user has agreed to allow open access to their accounts and data. The reading of the data is done in compliance with each site's terms and conditions. If an individual on the site has indicated they wish their information to be private, ASI respects the wish. Privacy is preserved using two methods: differential privacy and k-anonymity."

Differential privacy is a method used to processes personal information (e.g. age, gender, ethnicity) such that individual privacy is preserved: individuals are assigned probabilities for each demographic category and statistical noise is introduced so that it is not possible to determine the specific demographics of an individual [4]. The data is further protected using k-anonymity which removes demographic combinations that are below a small threshold to prevent against individuals being identified based on uncommon demographics. Having been highly successful in other prediction tasks such as correctly predicting the outcome of the Brexit referendum, the Trump election, and the Trudeau-led Liberal electoral victory in 2015 , the successes of this pilot project are highly anticipated.

For patients previously diagnosed with mental illness, researchers have turned to $\mathrm{Al} / \mathrm{ML}$ to assist physicians in treatment recommendation and modification. This is the focus of PhD Candidate Martin Cousineau's work at McGill University. Using medical health records from Douglas Mental Health University Institute in Montreal, he looks to assist physicians 
in achieving remission for patients suffering from treatment-resistant depression, a severe form of the major depressive disorder. His work has been used to identify the best initial treatment modification for incoming patients, recommend potential successful treatments, and characterize the current timing decisions between appointments. A variety of ML methods are leveraged in this work including the correction of selection bias introduced by the use of observational data, recommendation systems to personalize individual treatment (among hundreds of possible combinations), and inverse reinforcement learning to infer from the data the weights of multiple variables leveraged by a physician to select the date for the follow-up appointment. By no means trivial, when asked about the challenges in his work, Cousineau highlights the following:

\begin{abstract}
"The main challenge in my work consists in the limited suitable data available in comparison with the complexity of the task (e.g., predict the best treatment out of hundreds of possible combinations); there are many missing values which reduce the number of suitable observations. Another important challenge regarding my work pertains to using observational data that may be subject to a selection bias. While methods exist to reduce the effect of this bias, results generally require confirmation with randomized controlled trials (RCTs). My work can however suggest meaningful RCTs to run."
\end{abstract}

Despite these obstacles, Cousineau forges forward. His work promises to increase the efficiency of treatment management and ultimately, has the potential to save lives.

Research into utilizing multitudinous digital signatures to address mental illness is still in its infancy and considerable work remains. With the rising adoption of technology, data availability to support these initiatives is anticipated to increase and both ASI and Martin Cousineau express a shared vision of the future where artificial intelligence will significantly improve healthcare and humankind as a whole.

\title{
References
}

[1] Powles, J., Hodson, H. (2017) Google DeepMind and healthcare in an age of algorithms. Health Technol (Berl), 7 (4), 35167. Available from: http://www.ncbi.nlm.nih.gov/pubmed/29308344.

[2] Zauderer, M. G., Gucalp, A., Epstein, A. S., Seidman, A. D., Caroline, A., Granovsky, S., et al. (2014) Piloting IBM Watson Oncology within Memorial Sloan Kettering's regional network. American Society of Clinical Oncology, e17653.

[3] Kirkup, K. (2017) Ottawa backs Artificial Intelligence Program Looking for Early Suicide Warning Signs. Available from: https://globalnews.ca/news/3942921/ottawa-backs-artificial-intelligence-program-looking-for-earlysuicide-warning-signs/.

[4] Dwork, C. C. (2006) Differential Privacy. In: Proceedings of the 33rd international conference on Automata, Languages and Programming - Volume Part II. Springer-Verlag, p. 112. Available from: http://link.springer.com/10.1007/11787006_1.

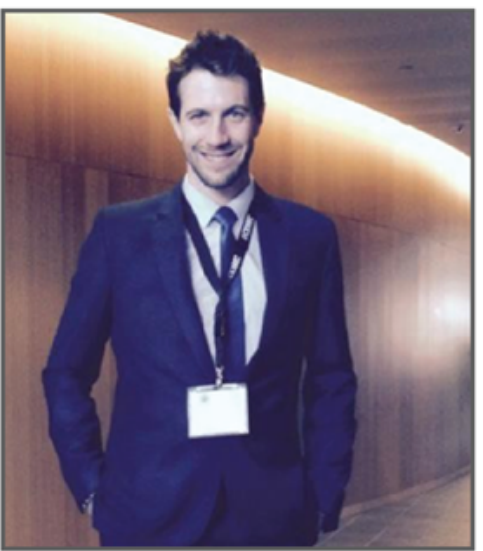

Kevin is a third year PhD candidate in Biomedical Engineering specializing in Data Science and Bioinformatics at Carleton University. He received his BSc in Biology and Computer Science from McGill University in 2014. His doctoral research focuses on improving protein-protein interaction prediction and leveraging context in related complete-graph problems such as protein function prediction and medical diagnosis prediction. As a polymath, Kevin spends his spare time exploring his curiosities at the intersection of the arts and sciences. 